\title{
Biological Effects of Atmospheric Fields and Radiation
}

\author{
P. P. Pathak, Awanish Sharma, Vijay Kumar
}

\begin{abstract}
Our atmosphere is full of electromagnetic fields emitted from wireless communication transmitters. At the same time the bio-systems (including humans, animals \& birds and vegetation as well) are complex electrical systems. Naturally therefore, they are likely to have interaction and to be affected in some way or the other. Research into this field started with the knowledge about pleasing presence of negative charges and unpleasant presence of positive charges around us, concentrating effects of static or pulsating fields. Also came the explanation (and experimental verification) of Hindu mythological effects of geomagnetic fields coupled with solar activity. The effects of power line radiation and radiation from video game $T V$ sets, microwave oven and other domestic instruments like electric blankets also figured in the research. At last the fastest emerging mobile telephony overpowered all others. Though the results of researches into possible harmful effects of mobile phone radiation and those of radiation from base station towers remain inconclusive partly because of influencing pressure from manufacturing and service providing companies, some of the researchers conclusively assert the harmfulness of the radiation at some level of field intensity present near the tower or the handset relating it to the specific absorption ratio (SAR) value. Recent increased observation of infertility among young couples is also attributed by some researchers to the effect of radiation from mobile kept in pantaloons pockets which becomes near their genitals affecting sperm or ovum. This review explains all these researches into bio-effects of electromagnetic fields and concludes that there is certainly some possible harmfulness of radiation above some level of intensity of fields.
\end{abstract}

Keywords: pantaloons pockets, harmfulness, communication transmitters.

\section{INTRODUCTION}

Due to uncontrolled explosion in number of man-made electromagnetic sources in last quarter of the 20th century and last 20 years of current century, our atmosphere became overflow with electromagnetic fields emitted from wireless communication transmitters. With the advancement of technologies, the intensity and frequency range is widening, resulting into more powerful fields. At the same time the bio-systems (including humans, animals \& birds and vegetation as well) are complex electrical systems. Naturally therefore, they are likely to have interaction and to be affected in some way or the other.

Revised Manuscript Received on August 10, 2019.

P. P. Pathak1, Professor, Department of Physics, Gurukula Kangri Vishwavidyalaya Haridwar, Uttrakhand, India. E-mail: pathakpp77@yahoo.com

Awanish Sharma, Professor, Department of Physics, Graphic Era Deemed to be University, Dehradun, Uttrakhand, India. E-mail: awanishiitr@gmail.com

Vijay Kumar, Professor, Department of Physics, Graphic Era Hill University, Dehradun, Uttrakhand, India. E-mail: vijay_phd05@rediffmail.com
Research into this field started with the knowledge about pleasing presence of negative charges and unpleasant presence of positive charges around us, concentrating effects of static or pulsating fields. Also came the explanation (and experimental verification) of Hindu mythological effects of geomagnetic fields coupled with solar activity (viz. lying with head towards north is harmful if solar activity is varied). The effects of power line radiation and radiation from video game TV sets, microwave oven and other domestic instruments like electric blankets also figured in the research. At last the fastest emerging mobile telephony overpowered all others. Though the results of researches into possible harmful effects of mobile phone radiation and those of radiation from base station towers remain inconclusive partly because of influencing pressure from manufacturing and service providing companies, some of the researchers conclusively assert the harmfulness of the radiation at some level of field intensity present near the tower or the handset relating it to the specific absorption ratio (SAR) value (Kottou et al. 2014, Kumar et al. 2015, Kaushik \& Pathak 2015, 2016, 2017a,b, Kaushik et al. 2017, 2018). Recent increased observation of infertility among young couples is also attributed by some researchers (Belpomme et al. 2018) to the effect of radiation from mobile kept in pantaloons pockets which becomes near their genitals affecting sperm or ovum.

This review explains all these researches into bio-effects of electromagnetic fields and concludes that there is certainly some possible harmfulness of radiation above some level of intensity of fields.

\section{OBSERVATIONAL FACTS}

(A) Static \& ELF fields: Presence of ions and static or slowly varying potentials were observed to affect the working of human beings (Persinger 1974). Some of the observations are as follows: Presence of positive ions gives laziness and the negative ones gives activeness and pleasure. Car driving concentration improved by putting $+1000 \mathrm{~V}$ on ceiling electrode along with $20 \mathrm{~V}$, $10 \mathrm{~Hz}$ AC.Similarly, $20 \mathrm{~V} / \mathrm{m}$ square pulse of $10 \mathrm{~Hz}$ gave Students more concentration. Radiation from high voltage power line was reported to cause blood coagulation and leukemia in the people living nearby.

An experiment to test the validity of Hindu mythological saying, "lying with head towards north is harmful" was carried out at Chennai, India. It was found that a person lying with head towards north felt fever and sweating when the alteration in magnetic field was done corresponding to solar disturbance. 


\section{Biological Effects of Atmospheric Fields and Radiation}

Same person with head towards south felt pleasant with same fluctuation (India Today Feb 1986). As short term effect Köning \& Ankermular (1960) reported the shortening of reaction time in presence of $2-6 \mathrm{~Hz}$ pulse of $5 \mathrm{~V}$ and it was lengthened in presence of $10 \mathrm{~Hz}$ pulse of same strength. Long term effect was observed by Wever (1974) that circadian rhythms were disturbed due to long exposure by $10 \mathrm{~Hz}$ pulse. It shortened the period of biological rhythms while it diminished the inter individual differences. But it also strengthened the coupling between different rhythms.

Living in acclimatized like that for better concentration in studies or with negatively charged room resulted in thrombocyte adhesiveness i.e. trend to blood coagulation. 10 $\mathrm{Hz}$ pulse increased blood clotting tendency within $3 \mathrm{~h}$ (could bestopped by drugs).

In frequency range $50-100 \mathrm{~Hz}$, findings influenced by power generation companies. But many studies showed that people working in power house and those living below the high voltage power lines have increased risk of leukemia and brain tumors. Fields produced by TV and Video sets and those produced by high voltage power lines is observed to enhance childhood leukemia and cancer risks.

Honey bee whose hives were located under $765 \mathrm{kV}$ power line exposed to $0.6-4.5 \mathrm{kV} / \mathrm{m} \quad 64 \%$ increases in overwinter mortality rate as comparedto shield hives at the same location. Those hives exposed to 1.1 to $8.3 \mathrm{kV} / \mathrm{m}$ showed almost no honey production and showed loss of beeweight. None of them survived next winter. Monkey trained to estimate time interval of $5 \mathrm{~s}$ exposed to $7 \mathrm{~Hz}$ field at 10 $\mathrm{V} / \mathrm{m}$ underestimated time interval. There are some useful effects like pacemaker for defective hearts and stimulation application of bias. First -ve bias is applied to suppresses infection but simultaneously growth is also suppressed. Then +vebias is applied which stimulates growth \& recovery. Plant growth is also affected by presence of fields or ions. Upward field enhances the growth while downward field restricts growth.

(B) VLF(3 - $30 \mathrm{kHz})$, LF(30 - $300 \mathrm{kHz}) \&$ MF(300 kHz - 3 MHz): No Effects have been reported to have been observed yet.

(C) HF (3 - $30 \mathrm{MHz}$ ) and higher frequency:Radio \& TV transmitters: According to Rai (1992) whole population around TV transmitter of Varanasi was affected. The effect reduced with increasing distance. This work is more of statistical nature than scientific.

\section{EFFECT AT DNA, CHROMOSOMAL, MICRONUCLEI AND SISTER CHROMATID LEVEL:}

Vijayalaxmi \& Obe (2005) gave a review of previous work on DNA Strand breaks, chromosomal aberration, micronuclei and sister chromatid exchanges due to Extremely Low Frequency (ELF) radiation during the period of $1990-2003$. They found that out of 63 studies, 29 (46\%) negative, $14(23 \%)$ positive and $20(32 \%)$ inconclusive. They also summarized similar studies of the same period for effect of radio frequency radiation (RFR) on this level of biological systems. In this they found that out of 53 Studies, $31(58 \%)$ showednegative results, $12(23 \%)$ showed positive for epilepsy. Bone growth and healing of wounds is by

results and10 (19\%) were inconclusive. It may be interesting to quote a discussion during XXVIII General Assembly at NPL New Delhi on these results presented there. One German scientists says, ' $23 \%$ results quoted to be positive is very large, even 1case positive in a million cases should be taken seriously'. Another (Indian) scientist (i.e. me) said that bodies of every individual have different immunities and one does not know about his immunity towards the radiation damage and these effects are likely to be irreparable. Therefore, the problem should be taken seriously.

\section{NATAL AND POST-NATAL INFLUENCES}

There is increasing evidence of EM radiation affects the developmental process of embryo inside the mother's womb. Othman et al.(2017) found that the in-utero exposure of mother to even the radiation from WiFi affected neurologically the offspring of rats as shown by disturbance in neurodevelopment, cerebral stress equilibrium and cholinesterase activityin them.Belpomme et al. (2018) are also of the view that 'there is strong evidence of increase in the risk of brain cancer' by long exposure of mobile phone radiation. They put 'the mechanism responsible includes the induction of reactive oxygen species, gene expression alteration and DNA damage through both epigenetic and genetic processes'. The generation of reactive oxygen species is held responsible for adverse effects on both male and female reproduction processes. Developments of electro-hypersensitivity or microwave illness are also attributed to neurobehavioral decrement caused by exposure.

\section{THE MECHANISM}

The electromagnetic field produced by transmitting antenna is propagated and fallson surface of a biological body coming across it. At a distance $r$ from a vertical short rod antenna radiating at power $\mathrm{P}$ the electric field is given by (Pathak et al. 2010)

$$
E_{r m s}=\frac{\sqrt{90 P}}{r}
$$

On penetration into a biological body the induced field at a depth $z$ from the surface is given by (Polk 1996)

$$
E_{i}=E_{r m s} \exp \left(\frac{-z}{\delta}\right)
$$

where the skin depth

$$
\delta=\frac{1}{\omega \sqrt{\frac{\varepsilon \mu}{2}\left[\left(1+p^{2}\right)^{1 / 2}-1\right]}}
$$

with $p=\sigma / \varepsilon \omega, \sigma$ being electrical conductivity, $\varepsilon$ the permittivity of the material of the biological body and $\omega$ being the radian frequency of the radiation. Radiation falling on human body comes across skin, blood,

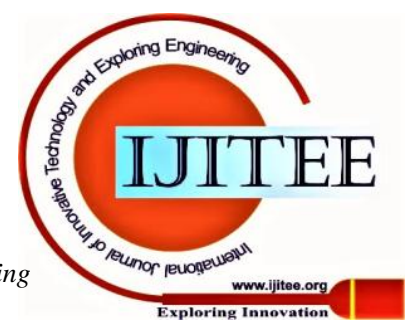


muscle and bone whose conductivity and permittivity are different and they also vary with frequency of radiation. Therefore, the skin depth $\delta$ also varies for different tissues as well aswith frequency of radiation. Thus electric component of electromagnetic field induced at different type of tissues will be different, in accordance with their conductivity, permittivity, depth inside the surface of the body even for a particular frequency of radiation. On calculation of field inside the body it is seen that it goes on reducing in magnitude. This reduction is linked with heating of tissues. In other words, one can say that the field is absorbed in the tissues. Thus absorption is calculated as specific absorption rate(SAR) given by (Adair \& Peterson 2002)

$S A R=\frac{\sigma E_{i}}{\rho}$

where $\rho$ is mass density of the material of tissue, $E_{i}$ the incident electric field intensity on it. Maximum permissible limitgiven by International Commission on Non - ionizing Radiation Protection (ICNRP) is $0.08 \mathrm{~W} / \mathrm{kg}$, which occurs at about $50 \mathrm{~m}$ from an antenna of $20 \mathrm{~W}$ radiating at $900 \mathrm{MHz}$. Due to the absorption of the fields, increase $\Delta T$ in temperature of the tissue within time $\Delta t$ in terms of specific heat $C$ is given by (Stuchly\&Stuchly 1990)

$$
C \Delta T=\operatorname{SAR}(\Delta t)
$$

Though this increase in temperature is modified by thermoregulation of the body. But it results in extra stress on the whole body which is likely to give many complications to the body.

Solution: Transmitters are to be installed in such a way that no person resides within at least $300 \mathrm{~m}$ of a $10 \mathrm{~kW}$ $\mathrm{TV} /$ Radio transmitter and at least $100 \mathrm{~m}$ from mobile phone tower.

\section{EFFECT OF MOBILE PHONE RADIATION ON OUR BODY:}

Generally mobile phones have $2 \mathrm{~W}$ antenna for receiving and transmitting the signals. The electric field at $2 \mathrm{~cm}$ from cell phone antenna radiating at $2 \mathrm{~W}$ comes out to be

\section{$\mathrm{E} \sim 14 / 0.02=700 \mathrm{~V} / \mathrm{m}$}

This will give about $650 \mathrm{~V} / \mathrm{m}$ incident on the brain after crossing through skull \& sculp. SAR and resulting temp. change can also be calculated. Similarly, for all the body parts resulting SAR can be calculated and it is seen that keeping the mobile phone near the body, the concerned body part is affected (Kumar et al. 2008, Sandeep \& Pathak, 2011). Keeping the mobile phone in the pocket of jeens/pantloons may become hazardous for young persons to affect their fertility.

Solution: Mobile sets should be kept about a meter away from the body. For carrying, it should be kept in purse or hand, not in pocket.

Conclusion: Harmfulness of mobile phone radiation and of that from base station towers is well establishedbeyond certain level of intensity, i.e. within certain distance from source depending on the power of transmitter. Department of Telecommunication, Government of India has issued guidelines for installation of base station transmitters and also for mobile phone users. Now it is our duty to check the installations of base station towers and report to the authorities if they are not in accordance of the guidelines. Also it is very important to follow the guidelines given for users so that it does not become harmful to us. Some important guidelines are as follows:

1. Keep cell phone farthest possible away from your head and other delicate parts of your body.

2. Use ear-phone for long conversation.

3. Do not bring phone near the ear, while other side it is ringing. Switch on loud speaker.

4. Do not keep switched on cell phone on your bed. Better to keep on a chair or stool about ameter away from your head.

5. Do not attend call with wet hair. Avoid using phone with low battery.

\section{REFERENCES}

1. Adair E R \& Peterson R C, Biological effects of radio frequency radiation, IEEE Trans micro theory technology, 50, 953, 2002.

2. Belpomme D, Hardell L, Belyaev I, Burgio E \& Carpenter D O, Thermal and non-thermal health effects of low intensity non-ionizing radiation: An international perspective, Environmental Pollution, 242, 643, 2018.

3. Kaushik Rahul \& Pathak P P, Effect of mobile phone radiation on human body,Int. J Sci. Engg. and Tech., 3(4), 863, 2015. (DOI10.2348/ijset07150863).

4. Kaushik Rahul \& Pathak P P, Estimation of specific absorption rate for $2 \mathrm{G}$ and $3 \mathrm{G}$ GSM frequency spectrum on human skin, Int. J. Sci. Engg. Tech., 4(3), 513, 2016

5. Kaushik Rahul \& Pathak P P, Specific absorption rate for human skin due to radiation of cylindrical wave front from transmission tower,Asian. J. Phys., 26(8-10), 309, 2017.

6. Kaushik Rahul, Kumar V \& Pathak P P, Variation of SAR with input power of cell phone antenna, J. Env. Bio. Sci., 31(2), 503, 2017.

7. Kaushik R, Kumar V, Teotia P\& Pathak P P, Variation of specific absorption rate in human head due to cylindrical wave front from vertical antenna of base transceiver station, IndianJournal of Environmental Protection,38 (1), 43, 2018.

8. Kotto S, Nikolopoulos D, Vogiannis E, Koulougliotis D, Petraki E \& Yannakopoulos P H, How Safe is the Environmental Electromagnetic Radiation? Physical Chemistry \& Biophysics, 4(3), 146, 2014.

9. Kumar V, Vats R P, Goyal S, Kumar S\& Pathak P P, Interaction of electromagnetic radiationwith human body, Indian J. Radio Space Phys. 37, 131, 2008.

10. Kumar S \& Pathak P P, Effect of electromagnetic radiation from mobile phones towers on human body, Indian J. Radio Space Phys. 40, 340, 2011.

11. Kumar A, Kumar V\& Pathak P P, Specific absorption rate (SAR) due to variation infrequency on human body tissues near radio broadcasting antenna, Asian J Pharmaceutical and Clinical Res., 8 , $120,2015$.

12. Othmana H, Ammaria M, Rtibic K, Bensaida N, Saklya M \&Abdelmeleka H, Postnatal development and behavior effects of inutero exposure of rats to radiofrequency waves emitted from conventional WiFi devices, Environmental Toxicology and Pharmacology, 52, 239, 2017.

13. Pathak P P, Tripathi H \& Kumar V,Specific absorption rate calculation and rate of temperature change in tissues due to radio antenna, Intl. Trans. In Appl. Sci. 2,709, 2010.

14. Persinger M A, ELF and VLF Electromagnetic Field Effects, Plenum Press, New Yark, 1974.

15. Polk C, Handbook of biological effects of electromagnetic Fields, (eds) C Polk, E Postow CRC Press, Boca Raton USA, 1996, pp 1-23.

16. Stuchly M A \&Stuchly S S, Biological effects of electromagnetic fields, Phys. Rev. (USA), 61, 435, 1981.

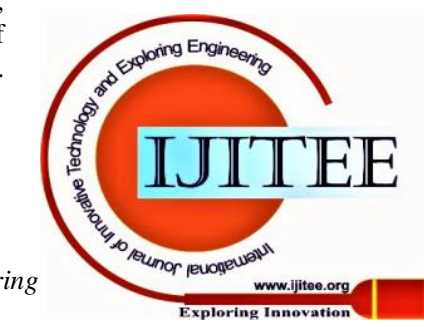

